\title{
A KERNEL COMPRESSION SCHEME FOR FRACTIONAL DIFFERENTIAL EQUATIONS*
}

\author{
DANIEL BAFFET ${ }^{\dagger}$ AND JAN S. HESTHAVEN ${ }^{\dagger}$
}

\begin{abstract}
The nonlocal nature of the fractional integral makes the numerical treatment of fractional differential equations expensive in terms of computational effort and memory requirements. In this paper we propose a method to reduce these costs while controlling the accuracy of the scheme. This is achieved by splitting the fractional integral of a function $f$ into a local term and a history term. Observing that the history term is a convolution of the history of $f$ and a regular kernel, we derive a multipole approximation to the Laplace transform of the kernel. This enables the history term to be replaced by a linear combination of auxiliary variables defined as solutions to standard ordinary differential equations. We derive a priori error estimates, uniform in $f$, and obtain estimates on the number of auxiliary variables required to satisfy an error tolerance. The resulting formulation is discretized to produce a time stepping method. The method is applied to some test cases to illustrate the performance of the scheme.
\end{abstract}

Key words. fractional differential equations, Volterra equations, kernel compression, local schemes

AMS subject classifications. 65R20, 45D05, 45G10, 45G15

DOI. $10.1137 / 15 \mathrm{M} 1043960$

1. Introduction. The nonlocal nature of fractional differential equations (FDEs) makes their numerical treatment considerably more difficult than that of standard differential equations. Direct approaches (e.g., $[1,2,3,4]$ and the references therein) for discretizing FDEs require that the entire solution history is stored and used throughout the computation. This may be expensive both in terms of computational and memory costs. In this paper we propose a method to address this difficulty.

Some methods for treating this issue have been proposed. It is worth mentioning the fixed memory principle [5] due to its simplicity. The use of nested meshes [6] has also been proposed to this end. Both these methods rely on storing past information and the direct evaluation of the convolution.

In this paper we take an approach more commonly used in the derivation of $a b$ sorbing or nonreflecting boundary conditions (see, e.g., [7] and the references therein). In this context, the method is designed to approximate the convolution

$$
w_{\delta} * f(t)=\int_{0}^{t} w_{\delta}(t-s) f(s) \mathrm{d} s
$$

by a linear combination of solutions $\psi_{1}, \ldots, \psi_{J}$ to initial value problems for standard ordinary differential equations (ODEs) of the form

$$
\psi^{\prime}=\lambda \psi+f, \quad \psi(0)=0,
$$

where $\lambda \in \mathbb{C}$. Here, $w_{\delta}(t)=w(t+\delta)$, and $w(t)=t^{-1+\alpha} / \Gamma(\alpha)$ is the kernel of the fractional integral. In the following we refer to $\psi_{1}, \ldots, \psi_{J}$ as the auxiliary variables of the scheme.

\footnotetext{
* Received by the editors October 14, 2015; accepted for publication (in revised form) December 9, 2016; published electronically March 7, 2017.

http://www.siam.org/journals/sinum/55-2/M104396.html

Funding: This work is partially funded by the National Science Foundation DMS-1115416.

$\dagger$ MATHICSE, École Polytechnique Fédérale de Lausanne (EPFL), 1015 Lausanne, Switzerland (daniel.baffet@epfl.ch, jan.hesthaven@epfl.ch).
} 
The idea to reduce the costs of evaluating the history term of the fractional integral [8], or more generally $[9,10,11]$ a convolution $K * f$, by approximating it by a linear combination of solutions to ODEs (1.2) has been explored in the past. In [8], this amounts to the approximation of the kernel $w$ by a linear combination of exponentials

$$
S(\Lambda, \sigma ; t)=\sum_{j=1}^{J} \sigma_{j} \mathrm{e}^{\lambda_{j} t}
$$

at some positive distance $\delta$ from the singularity. The kernel $w$ is approximated by a sum (1.3) with real nodes $\lambda_{j}$ and weights $\sigma_{j}$ on the interval $[\delta, \infty)$, where the estimate $|w-S| \leq \varepsilon$ of the absolute pointwise error holds for

$$
J=O\left((1-\alpha)^{-1}\left(\log (\alpha \varepsilon)^{-1}+\log \delta^{-1}\right)^{2}\right) .
$$

While the idea to approximate the kernel $w$ in the entire half line $t \geq \delta$ is appealing, it comes at the expense of the type of the error estimate possible. The reader may notice that a uniform estimate of the relative pointwise error of such approximation is impossible on $[\delta, \infty)$, as it requires the asymptotic behavior of a linear combination of exponentials to match the algebraic decay of $w$ as $t$ tends to infinity. The procedure in [12] also prescribes an approximation (1.3) with real nodes and weights. The authors discuss the approximation of some functions by sums of exponentials, including functions of the form $t^{-\beta}$. This approximation satisfies an estimate of the relative pointwise error $|w-S| \leq \varepsilon w$ in $[\delta, 1]$ for

$$
J=O\left(\left(\log \delta^{-1}+(1-\alpha)^{-1} \log \varepsilon^{-1}+\log \log \varepsilon^{-1}\right) \log \frac{1-\alpha}{\varepsilon}\right) .
$$

The algorithm presented in [9] has been used as the foundation for several methods [10, 13] and extended to allow variable step size [11]. To begin the discussion we outline the scheme proposed in [9]. The scheme is stated for the approximation of a convolution $K * f$, but we restrict the discussion to the problem of approximating the fractional integral. Suppose we wish to approximate the fractional integral of a function $f$ in $(0, T)$. Let $\delta>0$. The interval $[\delta, T]$ is covered by a set of geometrically growing, overlapping intervals $I_{\ell}$ with $\ell=1, \ldots, L$. In each $I_{\ell}$, the kernel $w$ is approximated by a sum $S_{\ell}$ of the form (1.3) obtained by the application of the trapezoidal rule to the inverse Laplace transform formula

$$
w(t)=\frac{1}{2 \pi i} \int_{\mathcal{C}_{\ell}} \mathrm{e}^{t \lambda} \widehat{w}(\lambda) \mathrm{d} \lambda,
$$

where $\widehat{w}$ is the Laplace transform of $w$. Each contour $\mathcal{C}_{\ell}$ is chosen $[11,14]$ so that the estimate $\left|w-S_{\ell}\right| \leq \varepsilon w$ holds in $I_{\ell}$ with $J=O\left(\log (\alpha \varepsilon)^{-1}\right)$ independent of $\ell$. As it takes $L=O\left(\log \delta^{-1} T\right)$ intervals $I_{\ell}$ to cover $[\delta, T]$, the total number of auxiliary variables required by this scheme to satisfy an error tolerance $\varepsilon$ in $[\delta, T]$ is $O\left(\log \delta^{-1} T \log (\alpha \varepsilon)^{-1}\right)$. Thus the approximation to the history term of the fractional integral of $f$ is given by a linear combination of auxiliary variables $\psi$ satisfying the ODE $\psi^{\prime}=\lambda \psi+f$ with different $\lambda \in \mathbb{C}$ and homogenous initial conditions at different times. The strength of this approach is that it offers a way to discard past values of $f$ and nonetheless account for their contribution efficiently. However, this approach results in complicated time stepping schemes, even when the step size $\Delta t=\delta$ remains constant. At each step, new auxiliary variables may be introduced, as $f$ is usually not known a priori, and old ones arded since they will not contribute to the procedure anymore. In addition, 
to be able to discard past values of $f$, at each step, all auxiliary variables must be advanced, but only some are actively used for the approximation of the history term.

Here, we take a different approach. We postpone describing the approximation to section 3 and in the following only discuss the properties of the method and the main result of the paper, given by Theorem 3.3. While for positive $\delta, w_{\delta}$ is smooth in $[0, \infty)$ and thus allows for the procedure, the singularity in the kernel $w=w_{0}$ of the fractional integral prevents the application of the method directly to $w_{0} * f=w * f$. As a consequence, the resulting approximation is not uniform in $\delta$. In the time stepping schemes below, we take $\delta$ to be the step size $\Delta t$, but for the sake of generality, we distinguish the two. For the proposed method to be cost effective, the number of auxiliary variables $J$ must be small. To measure this, it is natural to compare $J$ to the number of steps $N=T / \Delta t$. The analysis, however, is performed at the continuous level and provides uniform in $f$ error estimates. The main result of this paper, Theorem 3.3, yields the following a priori estimate of $J$ : Let $\mathcal{I}_{\delta}$ be the operator defined by $\mathcal{I}_{\delta} f=w_{\delta} * f$. For $T>0, \delta>0$, and an error tolerance $\varepsilon>0$, there exists an approximation operator $\mathcal{I}_{\delta, r}$, of the form $\mathcal{I}_{\delta, r} f=S * f$ with $J=p m$,

$$
p=O\left(\log \delta^{-1} T+\log \log \frac{1-\alpha}{\varepsilon}\right), \quad m=O\left(\log \varepsilon^{-1}\right),
$$

such that

$$
\left\|\mathcal{I}_{\delta} f-\mathcal{I}_{\delta, r} f\right\|_{L^{2}(0, T)} \leq \varepsilon\left\|\mathcal{I}_{\delta} f\right\|_{L^{2}(0, T)} \quad \forall f \in L^{2}(0, T) .
$$

Moreover, the analysis prescribes the approximation operator $\mathcal{I}_{\delta, r}$ explicitly, up to the evaluation of some integrals.

Thus, the scheme satisfies error estimates that show asymptotic behavior comparable to that of the estimates for the algorithm in [9]. The estimates are, however, on the relative error of the convolution approximation in the $L^{2}$-norm, rather than the relative pointwise error. Nevertheless, we obtain an estimate of the latter type as a corollary. We also note that the estimates, stated by Theorem 3.3, show that the number of poles $J$ required to satisfy a prescribed error tolerance is bounded uniformly for $\alpha \in(0,1)$. In fact, the estimates show that the error term associated with $p$ tends to zero when $\alpha$ tends to one. This is also demonstrated by our numerical results. While our estimates provide a uniform in $\alpha$ bound on the error term associated with $m$, in our numerical tests, near $\alpha=1$ we measure errors smaller than elsewhere. We emphasize that this is not reflected in our estimates and therefore we cannot conclude whether the error term associated with $m$ vanishes at the limit $\alpha \rightarrow 1$ or not. Nevertheless, the numerical results support the proposition that this term is also bounded uniformly for $\alpha \in(0,1)$.

In contrast to the methods inspired by [9], where different sets of auxiliary variables are used at different time "windows," here we use a single set of auxiliary variables. That is, in the entire time interval, the same set of auxiliary variables is used. This leads to relatively simple time stepping schemes. The kernel compression scheme also has features making it convenient for use with adaptive step size methods. In [15] we propose high order adaptive methods for FDEs based on the kernel compression scheme developed in this paper.

The rest of the paper is structured as follows. In section 2 we present an overview of the method, discuss its incorporation into a time stepping scheme and introduce some notation. The main results of the paper and their proofs are presented in section 3 , where we also state the approximation explicitly. In section 4 we provide details 
on the implementation of the kernel compression scheme and the fully discrete time stepping methods used in the numerical tests, whereas section 5 presents numerical results showing the approximation of the kernel $w_{\delta}$, and the application to two examples, including a fractional Van der Pol equation. We conclude with some remarks in section 6. Appendix A states and proves two lemmas on the multipole approximation and estimation of functions, and Appendix B provides some results on convolutions and the Laplace transform.

2. Overview. For $\alpha>0$, let

$$
\mathcal{I}^{\alpha} f(t)=\frac{1}{\Gamma(\alpha)} \int_{0}^{t}(t-s)^{-1+\alpha} f(s) \mathrm{d} s
$$

be the fractional integral of $f$. Consider $\mathcal{I}^{\alpha} f(t+\delta)$ for some $t \geq 0$ and $\delta>0$. We split the integral into a local term

$$
\int_{0}^{\delta} w(\delta-s) f(t+s) \mathrm{d} s
$$

and a history term

$$
\mathcal{I}_{\delta} f(t)=\int_{0}^{t} w_{\delta}(t-s) f(s) \mathrm{d} s
$$

where

$$
w_{\delta}(t)=w(t+\delta) \quad w(t)=\frac{t^{-1+\alpha}}{\Gamma(\alpha)} .
$$

In what follows we present the main idea of the method, propose an approach for incorporating it into a fully discrete time stepping scheme, and introduce the notation used subsequently.

2.1. Kernel compression. We denote by either $\widehat{f}$ or $\mathcal{L}[f]$ the Laplace transform

$$
\widehat{f}(\lambda)=\int_{0}^{\infty} \mathrm{e}^{-\lambda t} f(t) \mathrm{d} t
$$

of a function $f$. Let $\alpha \in(0,1), f:(0, T) \rightarrow \mathbb{R}^{d}$ and $\delta>0$. We may extend $f$ to $(0, \infty)$ by setting $f=0$ in $[T, \infty)$. Consider the history term $(2.3)$ of $\mathcal{I}^{\alpha} f(t+\delta)$, the fractional integral of $f$ at $t+\delta$. Its Laplace transform is given by

$$
\mathcal{L}\left[\mathcal{I}_{\delta} f\right](\lambda)=\widehat{w}_{\delta}(\lambda) \widehat{f}(\lambda) .
$$

As our goal is to approximate the convolution (2.3), we seek a multipole approximation

$$
r(\lambda)=\sum_{j=1}^{J} \frac{\sigma_{j}}{\lambda-\lambda_{j}}
$$

to $\widehat{w}_{\delta}$ that satisfies a uniform estimate of the relative error

$$
\left|\widehat{w}_{\delta}(\lambda)-r(\lambda)\right| \leq \varepsilon\left|\widehat{w}_{\delta}(\lambda)\right|, \quad \operatorname{Re} \lambda \geq \eta .
$$

The reason for requiring (2.8) is that it implies the following estimate of the relative 
$L^{2}$-error:

$$
\left\|\mathcal{I}_{\delta} f-\mathcal{I}_{\delta, r} f\right\|_{L^{2}(0, T)} \leq \varepsilon \mathrm{e}^{\eta T}\left\|\mathcal{I}_{\delta} f\right\|_{L^{2}(0, T)} \quad \forall f \in L^{2}(0, T),
$$

where $\mathcal{I}_{\delta, r} f$ is defined as the inverse Laplace transform of $r \widehat{f}$. Explicitly, $\mathcal{I}_{\delta, r} f$ is given by $\mathcal{I}_{\delta, r} f=S * f$, where

$$
S(t)=\sum_{j=1}^{J} \sigma_{j} \mathrm{e}^{\lambda_{j} t}
$$

is the inverse Laplace transform of $r$.

Given an approximation (2.7) to $\widehat{w}_{\delta}, \mathcal{I}_{\delta, r} f$ may also be expressed in terms of the solution to an initial value problem for a standard ODE system. Suppose $r$ is a multipole approximation $(2.7)$ to $\widehat{w}_{\delta}$. For each $j=1, \ldots, J$, define

$$
\widehat{\psi}_{j}=\left(\lambda-\lambda_{j}\right)^{-1} \widehat{f} .
$$

Inverting the Laplace transform we recover

$$
\psi_{j}(t)=\int_{0}^{t} \mathrm{e}^{\lambda_{j}(t-s)} f(s) \mathrm{d} s
$$

To simplify the notation we organize $\psi_{1}, \ldots, \psi_{J}$ as the columns of a matrix $\Psi=\left(\psi_{j}\right)$. Thus the approximation $\mathcal{I}_{\delta, r} f$ of $\mathcal{I}_{\delta} f$ associated with $r$ is

$$
\mathcal{I}_{\delta, r} f=\sum_{j=1}^{J} \sigma_{j} \psi_{j}=\Psi \sigma,
$$

where $\sigma=\left(\sigma_{1}, \ldots, \sigma_{J}\right)^{T}$. Observing that each $\psi_{j}$ is the solution to the ODE $\psi_{j}^{\prime}=$ $\lambda_{j} \psi+f$ satisfying $\psi_{j}(0)=0$, we recover

$$
\Psi^{\prime}=\Psi \Lambda+f 1, \quad \Psi(0)=0,
$$

where $\mathbf{1}=(1, \ldots, 1) \in \mathbb{R}^{J}$, and $\Lambda=\operatorname{diag}\left(\lambda_{1}, \ldots, \lambda_{J}\right)$. It is left to specify the multipole approximation $r$ to $\widehat{w}_{\delta}$ and derive the error estimate for the approximation $\mathcal{I}_{\delta, r} f$ of the history term $\mathcal{I}_{\delta} f$. These issues are addressed in section 3 .

2.2. Incorporation into a time stepping scheme. While the main focus of this paper is the approximation of (2.3), the goal of the method is to be incorporated into fully discrete time stepping schemes. In particular, schemes for initial value problems

$$
D^{\alpha} u=f(t, u), \quad u(0)=u_{0}
$$

in $(0, T)$, where $\alpha \in(0,1), f:[0, T] \times \Pi \rightarrow \mathbb{R}^{d}, T>0, \Pi \subset \mathbb{R}^{d}$ open, and $D^{\alpha}$ is the Caputo $\alpha$-derivative

$$
D^{\alpha} u=\mathcal{I}^{1-\alpha} u^{\prime}
$$

Below we propose a setup for the application of the kernel compression method as a part of a fully discrete time stepping scheme for (2.15). 
Applying $\mathcal{I}^{\alpha}$ to (2.15), we get

$$
u=u_{0}+\mathcal{I}^{\alpha}(f \circ u),
$$

where $f \circ u(t)=f(t, u(t))$. In fact, (2.15) and (2.17) are equivalent. A standard approach for the derivation of numerical methods for (2.17), and thus for (2.15), is as follows. Fix $t \geq 0$ and $\Delta t>0$, and let $\delta \in(0, \Delta t)$. Owing to (2.17), we have

$$
\begin{aligned}
u(t+\delta) & =\int_{0}^{\delta} w(\delta-s) f(t+s, u(t+s)) \mathrm{d} s+H(t, \delta), \\
H(t, \delta) & =u_{0}+\mathcal{I}_{\delta}(f \circ u)(t) .
\end{aligned}
$$

Observing that $(2.18 \mathrm{a})$ may be rewritten as

$$
U(\delta)=\mathcal{I}^{\alpha}(F \circ U)(\delta)+H(t, \delta),
$$

where $U(\delta)=u(t+\delta)$ and $F(\delta, u)=f(t+\delta, u)$, we find that two ingredients are required for the time stepping scheme. The first ingredient- the local time stepping scheme - is a method for approximating Volterra equations (2.19) on short intervals $(0, \Delta t)$, assuming $H$ is given. This scheme is applied to $(2.18 \mathrm{a})$ to advance the numerical solution from $t$ to $t+\Delta t$ and perhaps compute approximations of $u$ at a small number of points in $(t, t+\Delta t)$. In this paper we employ a one-step scheme of local order $2+\alpha$, but other schemes may also be viable. In practice, schemes approximating (2.19) require $H$ to be evaluated at some points in $(0, \Delta t]$. The computation of $H$ requires computing the history term and is therefore expensive to perform. To reduce the costs of evaluating the history term, we require the second ingredient of the scheme - the kernel compression scheme. This scheme prescribes the approximation in terms of the matrix of auxiliary variables $\Psi$, defined as the solution to (2.14). Thus we also need a method for approximating (2.14). For that purpose, we may use an A-stable method. The reason an A-stable method is required is that some of the $\lambda_{j}$-s have large negative real parts. In this paper we test the application of the trapezoidal rule and backward Euler scheme. Other schemes may also be used, e.g., A-stable diagonally implicit Runge-Kutta methods. Since $\Lambda$ is diagonal, this does not require solving a large algebraic system.

3. Kernel compression. To derive a rational approximation to $\widehat{w}_{\delta}$, we represent it as an integral of the form

$$
\widehat{w}_{\delta}(\lambda)=\int_{\mathcal{C}_{0}} \frac{\rho(\zeta)}{\lambda-\zeta} \mathrm{d} \zeta
$$

over a contour $\mathcal{C}_{0}$ in the complex plane and approximate this integral by some quadrature. Thus, different approximations may be obtained by choosing different contours and different quadratures. In the following we develop an error estimate for the approximation obtained with a specific integral representation of $\widehat{w}_{\delta}$ and the quadrature proposed by Lemma 3.5 of [7]. We rely on the following lemma which is inspired by that result. For the proof, see Appendix A.

Lemma 3.1. Let

$$
\phi(z)=\int_{\mathcal{C}} \frac{\rho(\zeta)}{z-\zeta} \mathrm{d} \zeta
$$

where $\mathcal{C}$ is a contour in the complex plane, and $\rho$ is integrable. Suppose $\mathcal{C} \subset \bigcup_{k=1}^{p} D_{k}$, where for each $k=1, \ldots, p, D_{k}$ is the closed disk of radius $r_{k}>0$ centered at $c_{k} \in \mathbb{C}$. 
Let

$$
r(z)=\sum_{k=1}^{p} \sum_{j=0}^{m-1} \frac{\sigma_{k j}}{z-\left(c_{k}+r_{k} \omega^{j}\right)},
$$

where $\omega=\mathrm{e}^{2 \pi i / m}$,

$$
\sigma_{k j}=\frac{1}{m} \sum_{l=0}^{m-1} \omega^{-j l} Q_{k l}, \quad Q_{k l}=\int_{\mathcal{C}_{k}} \rho(\zeta)\left(\frac{\zeta-c_{k}}{r_{k}}\right)^{l} \mathrm{~d} \zeta
$$

for each $k=1, \ldots, p, \mathcal{C}_{k}=\mathcal{C} \cap\left(D_{k} \backslash U_{k}\right)$, and $U_{k}=\bigcup_{j=1}^{k-1} D_{j}$. Suppose $a>1$, and $z \in \mathbb{C}$ satisfies $\left|z-c_{k}\right| \geq a r_{k}$ for each $k=1, \ldots, p$. Then, the following estimate holds:

$$
|\phi(z)-r(z)| \leq \frac{2}{a^{m}-1} \int_{\mathcal{C}}\left|\frac{\rho(\zeta)}{z-\zeta}\right| \mathrm{d}|\zeta|
$$

The error estimate for the approximation of $\widehat{w}_{\delta}$ is stated by Theorem 3.2 in section 3.2. As corollaries, we obtain the main results of the paper, Theorem 3.3 and Corollary 3.4 , which provide estimates in the time domain of the errors of the approximations to the convolution and the kernel, respectively. Both Theorem 3.3 and Corollary 3.4 are stated in section 3.2, where an overview of their proofs is also provided. The proof of Theorem 3.2 is presented in section 3.3. The technical details of all the proofs are provided in the appendices.

3.1. The multipole expansion. In what follows, we state the multipole approximation $r$ to $\widehat{w}_{\delta}$ and discuss the idea of its derivation. The analysis of the paper relies on properties of the upper incomplete gamma function [16, 17]. For convenience, we state these results without further reference. The Laplace transform $\widehat{w}_{\delta}$ of $w_{\delta}$ is given by

$$
\begin{aligned}
\widehat{w}_{\delta}(\lambda) & =\int_{0}^{\infty} \mathrm{e}^{-\lambda t} w(t+\delta) \mathrm{d} t=\frac{\mathrm{e}^{\delta \lambda}}{\Gamma(\alpha)} \int_{\delta}^{\infty} \mathrm{e}^{-\lambda t} t^{-1+\alpha} \mathrm{d} t \\
& =\lambda^{-\alpha} \mathrm{e}^{\delta \lambda} \frac{\Gamma(\alpha, \delta \lambda)}{\Gamma(\alpha)}
\end{aligned}
$$

where $\Gamma(\alpha, z)$ is the upper incomplete gamma function,

$$
\Gamma(\alpha, z)=\int_{z}^{\infty} \mathrm{e}^{-s} s^{\alpha-1} \mathrm{~d} s .
$$

Owing to

$$
\Gamma(\alpha, z)=\frac{z^{\alpha} \mathrm{e}^{-z}}{\Gamma(1-\alpha)} \int_{0}^{\infty} \frac{x^{-\alpha} \mathrm{e}^{-x}}{z+x} \mathrm{~d} x,
$$

valid for $\alpha \in(0,1)$ and $\operatorname{Re} z>0$, we recover

$$
\widehat{w}_{\delta}(\lambda)=K_{\alpha} \int_{0}^{\infty} \frac{x^{-\alpha} \mathrm{e}^{-\delta x}}{\lambda+x} \mathrm{~d} x
$$

where

$$
K_{\alpha}=\frac{1}{\Gamma(\alpha) \Gamma(1-\alpha)}=\frac{\sin (\pi \alpha)}{\pi} .
$$




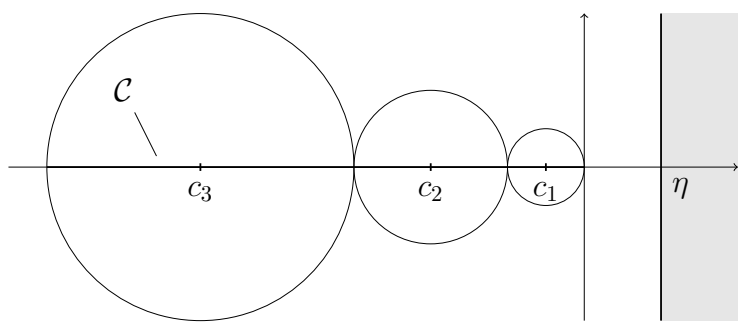

FIG. 1. The setup in the complex plane: the half plane $\operatorname{Re} z \geq \eta$ and the contour $\mathcal{C}$ covered by the disks $D_{k}$ centered at $c_{k}$ of radii $r_{k}$.

Let $\alpha \in(0,1), \eta>0$, and $p, m \in \mathbb{N}$. The resulting expansion is given by

$$
r(\lambda)=\sum_{k=1}^{p} \sum_{j=0}^{m-1} \frac{\sigma_{k j}}{\lambda-\lambda_{k j}}, \quad \lambda_{k j}=c_{k}+r_{k} \omega^{j},
$$

where $\omega=\mathrm{e}^{2 \pi i / m}$, for each $k=1, \ldots, p$ and $j=0, \ldots, m-1$,

$$
\begin{aligned}
\sigma_{k j} & =\frac{1}{m} \sum_{l=0}^{m-1} \omega^{-j l} Q_{k l}, \\
Q_{k l} & =K_{\alpha} \int_{x_{k-1}}^{x_{k}} x^{-\alpha} \mathrm{e}^{-\delta x}\left(\frac{-x+\left|c_{k}\right|}{r_{k}}\right)^{l} \mathrm{~d} x, \\
r_{k} & =\eta 2^{k-2} \quad c_{k}=-\eta\left(3 \cdot 2^{k-2}-1\right),
\end{aligned}
$$

and

$$
x_{k}(\eta)=\eta\left(2^{k}-1\right), \quad k=0, \ldots, p .
$$

The general idea of the derivation of the approximation is as follows. For $\eta>0$ and $p \in \mathbb{N}$ we approximate (3.8) by an integral over the truncated interval $\left(0, x_{p}\right)$. To be consistent with the spirit of the proof of Theorem 3.2, we treat the resulting integral as a contour integral over the segment $\left(-x_{p}, 0\right)$, directed from $\zeta=0$ to $\zeta=-x_{p}$. Thus, we apply Lemma 3.1 to approximate that contour integral. We divide the integral over $\left(-x_{p}, 0\right)$ into a sum of integrals over the disjoint intervals $I_{k}=\left(-x_{k},-x_{k-1}\right)$ with $k=1, \ldots, p$. Note that for each $k=1, \ldots, p$, the center and radius of $I_{k}$ are $c_{k}$ and $r_{k}$, respectively. Finally, the approximation (3.10) is obtained by replacing, for each $k=1, \ldots, p$, the integral over $I_{k}$, by a sum of $m$ poles located on the circle of radius $r_{k}$ centered at $c_{k}$. Figure 1 shows an illustration of the setup in the complex plane.

3.2. Statement of main results. The main results are stated below. Theorem 3.2 provides an error estimate for the approximation of the Laplace transform of the kernel.

Theorem 3.2. Let $\alpha \in(0,1), \eta>0$, and $\delta>0$. Then, $r$ given by (3.10) satisfies

$$
\left|\widehat{w}_{\delta}(\lambda)-r(\lambda)\right| \leq C_{m p}(\alpha, \delta \eta)\left|\widehat{w}_{\delta}(\lambda)\right|
$$

for all $\operatorname{Re} \lambda \geq \eta$, where

$$
C_{m p}(\alpha, \eta)=C_{a} A_{m}+C_{b} B_{p}(\alpha, \eta)
$$


with

$$
A_{m}=3^{-m}, \quad B_{p}(\alpha, \eta)=\frac{\Gamma\left(1-\alpha, \eta\left(2^{p}-1\right)\right)}{\Gamma(1-\alpha)} .
$$

The constants $C_{a}$ and $C_{b}$ are positive and independent of $\alpha, \eta, \delta, p$, and $m$.

The reader is referred to section 3.3 for the proof. While the term $B_{p}$ in $(3.12 \mathrm{~b})$ seems to describe the error very well (see the numerical results in section 5.1), it may not be convenient for use in applications, as it relies on the incomplete gamma function. In that case, one may consider using the inequality

$$
B_{p}(\alpha, \eta) \leq C(1-\alpha) x_{p}^{-\alpha}(\eta) \mathrm{e}^{-x_{p}(\eta)},
$$

which captures the asymptotic behavior of $B_{p}$ for large $p$, to estimate the error.

Owing to Theorem 3.2, we have the following estimate of the global error of the convolution approximation.

Theorem 3.3. Let $\alpha \in(0,1), T>0, \eta>0, \delta>0$, and $m, p \in \mathbb{N}$. Suppose $r$ is given by (3.10), and $\mathcal{I}_{\delta, r}$ is the approximation operator associated with $r$, i.e., $\mathcal{I}_{\delta, r} f=S * f$, where $S=\mathcal{L}^{-1}[r]$. Then, the estimate

$$
\left\|\mathcal{I}_{\delta} f-\mathcal{I}_{\delta, r} f\right\|_{L^{2}(0, T)} \leq \mathrm{e}^{\eta T} C_{m p}(\alpha, \delta \eta)\left\|\mathcal{I}_{\delta} f\right\|_{L^{2}(0, T)}
$$

holds for every $f \in L^{2}(0, T)$.

Proof. Estimate (3.14) follows immediately from Lemma B.2. The hypotheses of that lemma hold due to Theorem 3.2, (3.5), and Lemma A.1, which implies that $\widehat{w}_{\delta}$ has no zeros in the right half plane.

As a corollary to Theorem 3.3, we obtain an estimate of the relative pointwise error of the kernel approximation; an estimate of the type obtained for the methods in $[12,14]$. This estimate follows from (3.14) and Lemma B.3, as $w_{\delta}$ and $S$ are continuous on $[0, \infty)$. Thus, we have the following.

Corollary 3.4. Let $\alpha \in(0,1), T>0, \eta>0, \delta>0$, and $m, p \in \mathbb{N}$. Then $S=\mathcal{L}^{-1}[r]$, where $r$ is given by (3.10), satisfies the estimate

$$
\left|w_{\delta}(t)-S(t)\right| \leq \mathrm{e}^{\eta T} C_{m p}(\alpha, \delta \eta)\left|w_{\delta}(t)\right|
$$

holds for all $t \in[0, T]$.

3.3. Proof of Theorem 3.2. It is convenient to perform the analysis on the Laplace transform

$$
\widehat{v}(z)=\delta^{-\alpha} \widehat{w}_{\delta}\left(\delta^{-1} z\right)=z^{-\alpha} \mathrm{e}^{z} \frac{\Gamma(\alpha, z)}{\Gamma(\alpha)}
$$

of

$$
v(t)=w_{1}(t)=\delta^{1-\alpha} w_{\delta}(\delta t)=\frac{1}{\Gamma(\alpha)}(1+t)^{-1+\alpha}
$$

and deduce the conclusion for $\widehat{w}_{\delta}$ at the end. Observing that $\Gamma(\alpha, z) / \Gamma(\alpha)=1-$ $z^{\alpha} \gamma^{*}(\alpha, z)$, where $\gamma^{*}$ is an entire function, we find that $\widehat{v}(z)$ is given by

$$
\widehat{v}(z)=\left(z^{-\alpha}-\gamma^{*}(\alpha, z)\right) \mathrm{e}^{z}
$$

and is therefore analytic and single valued in $\mathbb{C} \backslash(-\infty, 0]$. To obtain a rational 
approximation to $\widehat{v}$ we rely on the representation (3.7) of the incomplete gamma function which yields

$$
\widehat{v}(z)=K_{\alpha} \int_{0}^{\infty} \frac{x^{-\alpha} \mathrm{e}^{-x}}{z+x} \mathrm{~d} x .
$$

For the approximation of (3.18) we use the quadrature proposed by Lemma 3.5 of [7]. Although different, the lemma used in this paper, Lemma 3.1, is inspired by that result. The proof of Theorem 3.2 relies on a similar result for $\widehat{v}$, given by Lemma 3.6 below, which requires the following proposition.

Proposition 3.5. Let $\alpha \in(0,1)$ and

$$
V_{1}(a, z)=K_{\alpha} \int_{0}^{a}\left|\frac{x^{-\alpha} \mathrm{e}^{-x}}{z+x}\right| \mathrm{d} x, \quad V_{2}(a, z)=K_{\alpha} \int_{a}^{\infty}\left|\frac{x^{-\alpha} \mathrm{e}^{-x}}{z+x}\right| \mathrm{d} x
$$

for $a \in[0, \infty)$ and $0 \neq z \in \mathbb{C}$ with $\operatorname{Re} z \geq 0$. There exist constants $C_{0}, C_{1}$, and $C_{2}$, independent of $\alpha$ such that for all $a \geq 0$ and $0 \neq z \in \mathbb{C}$ with $\operatorname{Re} z \geq 0$, the following estimates hold:

$$
\begin{aligned}
\widehat{v}(|z|) & \leq C_{0}|\widehat{v}(z)|, \\
V_{1}(a, z) & \leq C_{1}|\widehat{v}(z)|, \\
V_{2}(a, z) & \leq C_{2} \frac{\Gamma(1-\alpha, a)}{\Gamma(1-\alpha)}|\widehat{v}(z)| .
\end{aligned}
$$

Proof. Estimate (3.20) follows readily from (3.18) and Lemma A.1. To prove (3.21) and (3.22), it suffices to show they hold for $z=\mu$ real and positive, due to (3.20), (3.18), and Lemma A.1. For $z=\mu>0,(3.21)$ is trivial. Thus, it is left to show (3.22) with $z=\mu>0$. Specifically, we show (3.22) with $z=\mu>0$ and $C_{2}=1$, or equivalently, we show that

$$
\Delta(a, \mu)=\Gamma(1-\alpha, a) \widehat{v}(\mu)-\Gamma(1-\alpha) V_{2}(a, \mu)
$$

is nonnegative. Observing that for $\mu>0, \widehat{v}(\mu)=V_{1}(a, \mu)+V_{2}(a, \mu)$, and $\Gamma(1-\alpha)=$ $\Gamma(1-\alpha, a)+\gamma(1-\alpha, a)$, where $\gamma$ is the lower incomplete gamma function, we recover

$$
\Delta(a, \mu)=\Gamma(1-\alpha, a) V_{1}(a, \mu)-\gamma(1-\alpha, a) V_{2}(a, \mu),
$$

after the cancellation of terms. Explicitly, we have

$$
\Delta(a, \mu)=\int_{0}^{a} \int_{a}^{\infty} \frac{y-x}{(\mu+x)(\mu+y)}(x y)^{-\alpha} \mathrm{e}^{-(x+y)} \mathrm{d} y \mathrm{~d} x,
$$

and the conclusion follows.

We are now in a position to prove the error estimate for the multipole approximation to $\widehat{v}$.

Lemma 3.6. Let $\alpha \in(0,1)$ and $\eta>0$. Then, $r$ given by (3.10) with $\delta=1$ satisfies

$$
|\widehat{v}(z)-r(z)| \leq C_{m p}(\alpha, \eta)|\widehat{v}(z)|
$$

for all $\operatorname{Re} z \geq \eta$, where $C_{m p}$ is given by (3.12), and the constants $C_{a}$ and $C_{b}$ are positive and independent of $\alpha, \eta, p$, and $m$. 
Proof. The proof is based on the integral representation (3.18) of $\widehat{v}$ and Lemma 3.1. We begin by sketching the construction of the multipole approximation and the argument justifying the application of Lemma 3.1. We refer to Figure 1 for an illustration of the setup. For $p \in \mathbb{N}$, define $x_{p}=x_{p}(\eta)=\eta\left(2^{p}-1\right)$ and

$$
\widehat{v}_{1}(z)=K_{\alpha} \int_{0}^{x_{p}} \frac{x^{-\alpha} \mathrm{e}^{-x}}{z+x} \mathrm{~d} x, \quad \widehat{v}_{2}(z)=K_{\alpha} \int_{x_{p}}^{\infty} \frac{x^{-\alpha} \mathrm{e}^{-x}}{z+x} \mathrm{~d} x .
$$

Note that $\widehat{v}_{1}$ has the form

$$
\widehat{v}_{1}(z)=\int_{\mathcal{C}} \frac{\rho(\zeta)}{z-\zeta} \mathrm{d} \zeta
$$

where $\rho(\zeta)=-K_{\alpha}(-\zeta)^{-\alpha} \mathrm{e}^{\zeta}$, and the contour $\mathcal{C}$ is the segment $\left(-x_{p}, 0\right)$ in the complex plane directed from $\zeta=0$ to $\zeta=-x_{p}$. Also note that $\mathcal{C}$ is covered by the disks

$$
D_{k}=\left\{\zeta \in \mathbb{C}:\left|\zeta-c_{k}\right| \leq r_{k}\right\}, \quad k=1, \ldots, p,
$$

where $r_{k}$ and $c_{k}$ are given by (3.10d). The reader may verify that for each $k=1, \ldots, p$ and $z \in \mathbb{C}$ with $\operatorname{Re} z \geq \eta$, there holds $\left|z-c_{k}\right| \geq 3 r_{k}$. By Lemma 3.1, (3.10) with $\delta=1$ satisfies

$$
\left|\widehat{v}_{1}(z)-r(z)\right| \leq \frac{2}{3^{m}-1} V_{1}\left(x_{p}, z\right)
$$

for all $\operatorname{Re} z \geq \eta$, where $V_{1}$ is given by (3.19). By $\widehat{v}=\widehat{v}_{1}+\widehat{v}_{2}$, the triangle inequality, (3.29), and

$$
\left|\widehat{v}_{2}(z)\right| \leq V_{2}\left(x_{p}, z\right)
$$

where $V_{2}$ is given by (3.19), we have

$$
|\widehat{v}(z)-r(z)| \leq \frac{2}{3^{m}-1} V_{1}\left(x_{p}, z\right)+V_{2}\left(x_{p}, z\right),
$$

and thus Proposition 3.5 yields the conclusion.

This now allows us to complete the proof of Theorem 3.2.

Proof of Theorem 3.2. Suppose $\delta_{0}>0$. Let $\widetilde{\eta}=\delta_{0} \eta$. By Lemma 3.6, $\widetilde{r}$ given by (3.10) with $\delta=1$ satisfies (3.26) for all $\operatorname{Re} z \geq \widetilde{\eta}$. That is,

$$
\left|\widehat{v}\left(\delta_{0} \lambda\right)-\widetilde{r}\left(\delta_{0} \lambda\right)\right| \leq C_{m p}\left(\alpha, \delta_{0} \eta\right)\left|\widehat{v}\left(\delta_{0} \lambda\right)\right|
$$

holds for all $\operatorname{Re} \lambda \geq \eta$. We multiply (3.32) by $\delta_{0}^{\alpha}$ and use $\widehat{w}_{\delta_{0}}(\lambda)=\delta_{0}^{\alpha} \widehat{v}\left(\delta_{0} \lambda\right)$ to recover (3.11) with $\delta=\delta_{0}$ and $r(\lambda)=\delta_{0}^{\alpha} \widetilde{r}\left(\delta_{0} \lambda\right)$. Observing that $r$ may written in the form (3.10) with $\delta=\delta_{0}$, we have the conclusion.

4. Implementation. The scheme has two main components, comprising the kernel compression scheme to treat the time history and the local time stepping scheme. We discuss these separately in the following.

4.1. Kernel compression scheme. The scheme requires the weights $\sigma_{k j}$ and poles $\lambda_{k j}$ prescribed in (3.10). These parameters depend only on $\alpha, \eta$, and the distance 
from the kernel singularity $\delta$. In the time stepping methods tested below, $\eta=1 / T$, where $T$ is the simulation interval length, and $\delta=\Delta t$, where $\Delta t$ is the time step size $\Delta t$. Thus, provided the step size $\Delta t$ is constant, we may compute the weights and poles once and use them throughout.

While it is straightforward to compute the poles $\lambda_{k j}$, the computation of the weights $\sigma_{k j}$ deserves attention. Observing (3.10b), we note that for each $k=1, \ldots, p$, $\left(\sigma_{k j}\right)_{j}$ is the discrete Fourier transform of $\left(Q_{k l}\right)_{l}$ and thus may be computed efficientlyeven for large $m$, although this is not expected in practice. Computing $Q_{k l}$ requires the evaluation of the integrals (3.10c). In our implementation, each $Q_{k l}$ is approximated by an appropriate Gauss-Jacobi quadrature. In each interval $\left(x_{k-1}, x_{k}\right)$ with $k \geq 2$, the integrand of (3.10c) is smooth and thus (3.10c) may be approximated by a Gauss-Legendre quadrature. In $\left(x_{0}, x_{1}\right)$, however, the integrand has a singularity at the left endpoint $x_{0}=0$. We, therefore, employ the Gauss-Jacobi quadrature corresponding to the singularity for the approximation of (3.10c) with $k=1$. In the tests below we use quadratures with $4 m+1$ nodes.

The number $J=p m$ of auxiliary variables may be reduced in computations to about half. We exploit that the poles are distributed symmetrically around the real line to remove poles with negative imaginary parts and real duplicate poles to recover

$$
\sum_{k=1}^{p} \sum_{j=0}^{m-1} \sigma_{k j} \psi_{k j}=\operatorname{Re} \sum_{j=1}^{P} \theta_{j} \psi_{j}
$$

The procedure is outlined in Algorithm 1.

Algorithm 1 treats the cases where $m$ is even or odd differently. The resulting number of poles $P$ in the current implementation is given by $P=p m / 2+1$ if $m$ is even and $P=p(m+1) / 2$ if $m$ is odd. This has the following implication. Let $m$ be an odd number. The accuracy of the scheme is determined by the effective number of poles $J=p m$, and the computational cost is that of computing $P=p(m+1) / 2$ auxiliary variables. However, replacing $m$ by $m+1$ provides the accuracy obtained by $J_{1}=p(m+1)=J+p$ poles at the cost of computing only $P_{1}=p(m+1) / 2+1=$ $P+1$ auxiliary variables. That is, when $m$ is odd, the added computational cost of increasing $m$ by one is small compared to the potential added accuracy.

To avoid the appearance of different behaviors of errors with respect to $P$ and the switching between the two different algorithms (when $m$ is odd or even), in this paper, the numerical results are obtained with odd $m$.

4.2. Time stepping schemes. Consider the initial value problem (2.15). Let $N \in \mathbb{N}, \Delta t=T / N$, and $t_{n}=n \Delta t$ with $n=0, \ldots, N$. Below, $v$ and $\Phi=\left(\varphi_{j}\right)$ denote the numerical approximations to $u$ and $\Psi=\left(\psi_{j}\right)$, respectively, $\theta_{j}$ and $\lambda_{j}$, with $j=1, \ldots, P$, are obtained from applying Algorithm 1 to the weights $\sigma_{k j}$ and nodes $\lambda_{k j}$ given by (3.10) with $\delta=\Delta t$. Following the discussion in section 2.2, we approximate (2.18a) by

$$
v^{n+1}=\Delta t^{\alpha} \sum_{k=0}^{1} a_{k} f^{n+k}+H^{n}
$$

where $f^{n}=f\left(t_{n}, v^{n}\right)$, and

$$
a_{0}=\frac{\alpha}{\Gamma(2+\alpha)}, \quad a_{1}=\frac{1}{\Gamma(2+\alpha)}, \quad H^{n}=u_{0}+\operatorname{Re} \sum_{j=1}^{P} \theta_{j} \varphi_{j}^{n} .
$$




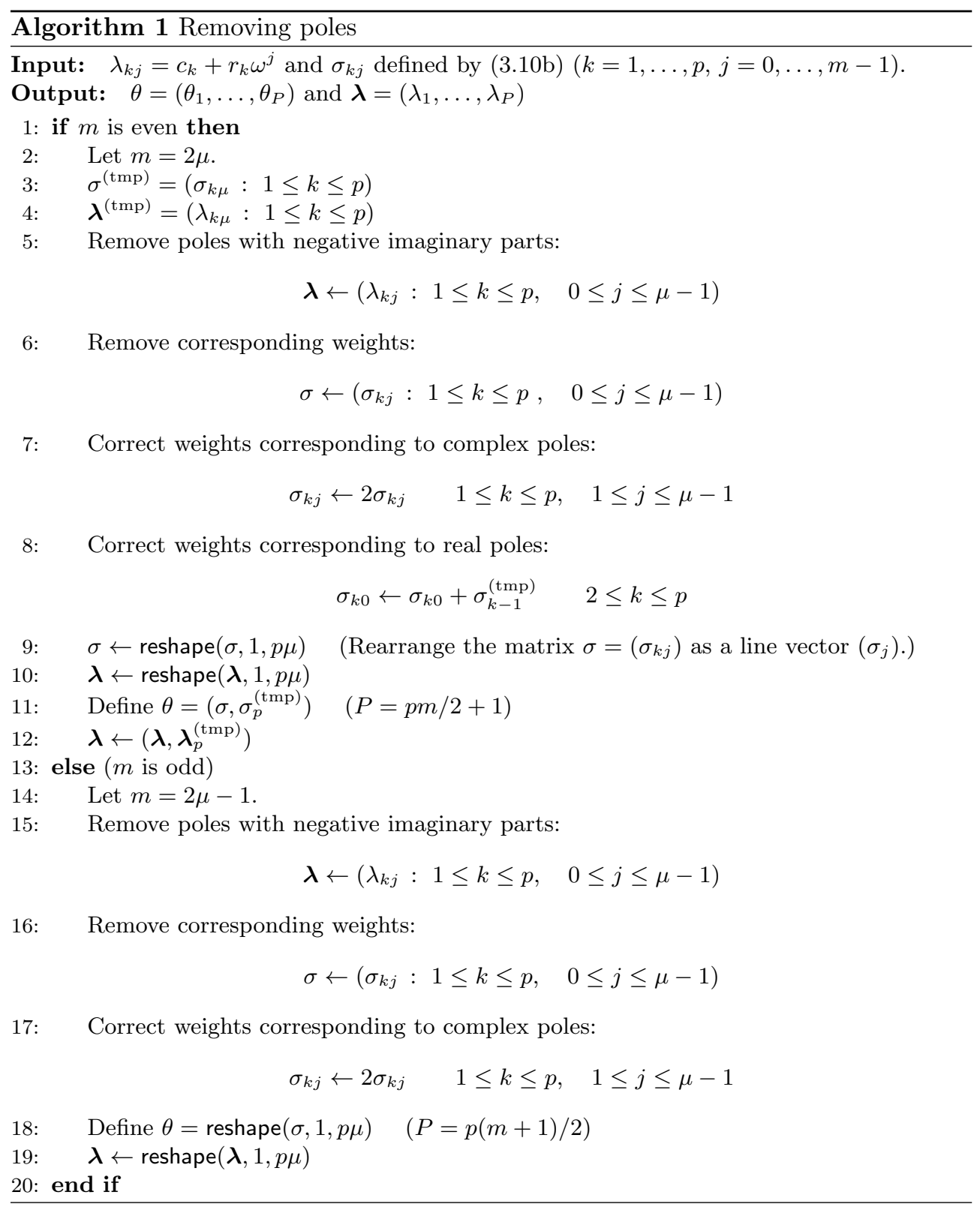

Note that (4.2) requires the solution of an algebraic equation; this is done by a Newton solver.

Since some of the poles $\lambda_{1}, \ldots, \lambda_{P}$ have large negative real parts, we use a onestep $A$-stable method to approximate (2.14). Note that coupling in (2.14) occurs only through $f$. Also note that $f$ is only a function of $v$ and that $v^{n+1}$ depends only on $\Phi^{n}$. Thus, applying one-step implicit schemes to (2.14) is simple. (Recall that $\Lambda$ is diagonal.) We test the application of a trapezoidal rule,

$$
\Phi^{n+1}=\left[\Phi^{n}\left(I+\frac{\Delta t}{2} \Lambda\right)+\frac{\Delta t}{2}\left(f^{n+1}+f^{n}\right) 1\right]\left(I-\frac{\Delta t}{2} \Lambda\right)^{-1},
$$



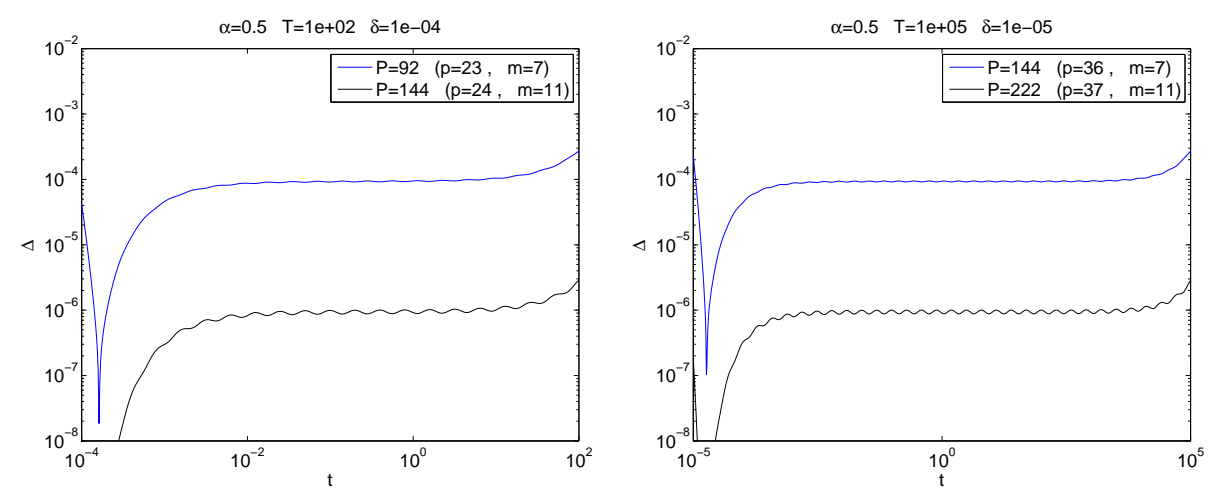

FIG. 2. The relative error $\Delta$ given by (5.1) as a function of $t$; results are obtained with $T=10^{2}$ and $\delta=10^{-4}$, on the left, and $T=10^{5}$ and $\delta=10^{-5}$, on the right.

and the backward Euler scheme,

$$
\Phi^{n+1}=\left(\Phi^{n}+\Delta t f^{n+1} \mathbf{1}\right)(I-\Delta t \Lambda)^{-1},
$$

where $\Lambda=\operatorname{diag}\left(\lambda_{1}, \ldots, \lambda_{P}\right)$, and $\mathbf{1}=(1, \ldots, 1) \in \mathbb{R}^{P}$. To advance the numerical solution from $t_{n}$ to $t_{n+1}=t_{n}+\Delta t$, the schemes above only require $v^{n}$ and $\Phi^{n}$. Given these values, we proceed by computing $v^{n+1}$ by (4.2). Now we may evaluate $f^{n+1}$ and compute $\Phi^{n+1}$ by either (4.4) or (4.5). Thus we obtain all the information required for another step. To initiate the procedure we set $\Phi^{0}=0$, owing to the initial condition in (2.14).

5. Numerical tests. In section 5.1 , we test the kernel compression scheme directly. Thus, the tests do not involve time stepping. The results in sections 5.2 and 5.3 are obtained using the time stepping methods described in section 4.2. In the following, $p$ is the number of circles, $m$ is the number of poles on each circle, and $P$ is the number of auxiliary variables used in the calculation, excluding unnecessary poles.

5.1. The kernel compression scheme. In this section we test the kernel compression scheme directly, without involving time stepping. Specifically, we test estimate (3.15). Thus, let

$$
\Delta(t)=\left|\frac{w(t)-S(t-\delta)}{w(t)}\right|, \quad S(t)=\operatorname{Re} \sum_{j=1}^{P} \theta_{j} \mathrm{e}^{\lambda_{j} t}
$$

be the relative error of the kernel approximation. The results are obtained with $\eta=1 / T$. Unless mentioned otherwise, $\alpha=1 / 2$. Figure 2 shows the relative error $\Delta$ as a function of $t$ in $[\delta, T]$ for approximations computed with different $p$ and $m$. The results are obtained for $T=10^{2}$ and $\delta=10^{-4}$ on the left, and $T=10^{5}$ and $\delta=10^{-5}$ on the right. The relative error seems to be bounded uniformly in $[\delta, T]$ as $(3.15)$ suggests.

In the following we fix $T=10^{2}$. Figure 3 shows

$$
M=\max _{n} \Delta\left(\tau_{n}\right)
$$



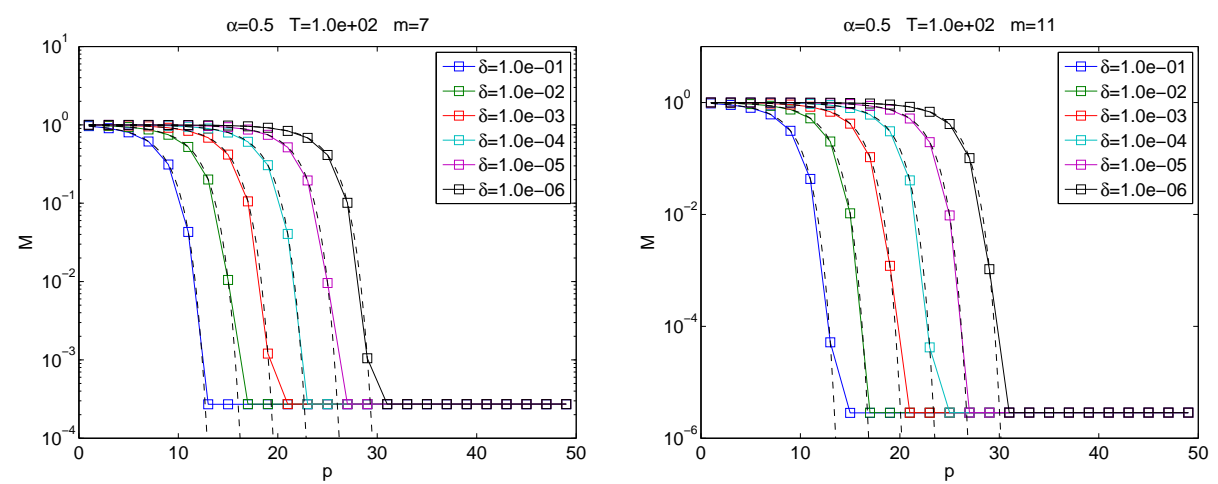

FIG. 3. The maximum value $M$, given by (5.3), of the relative error $\Delta$ on the grid $\tau_{n}$ as a function of $p$; the different graphs correspond to different values of $\delta$; results are obtained with $m=7$ on the left and $m=11$ on the right.

as a function of $p$, where for each $n=0, \ldots, N_{\text {samp }}, \tau_{n} \in\left[\delta, T+\delta 10^{q}\right]$ is given by

$$
\tau_{n}=\delta 10^{q n}, \quad q=10^{-2}, \quad N_{\mathrm{samp}}=\left\lceil\frac{\log \delta^{-1} T}{q \log 10}\right\rceil .
$$

The dashed lines are the graphs of $B_{p}(\alpha, \delta \eta)$, given by $(3.12 \mathrm{~b})$, indicating the theoretical estimate (3.15). Results on the left and right are obtained with $m=7$ and $m=11$, respectively, and the different graphs correspond to different $\delta$. We use $\tau_{n}$ given by (5.3) to cover the interval $[\delta, T]$ uniformly on a logarithmic scale. A similar grid is used for plotting Figure 2. Figure 3 shows that when $m$ is sufficiently large, the theoretical estimate predicts the error very well. The figure shows the rapid convergence in $p$ until the error reaches a value, which seems independent of $\delta$, where it levels. At that point the error is saturated by the term associated with $m$ and can only be reduced by increasing $m$. In particular, the results show that the number $m$ of poles on each circle required to achieve a given error tolerance is independent of $\delta$. This behavior is consistent with the theoretical estimate (3.15), as $A_{m}$, given by (3.12b), is independent of $\eta$ and $\delta$. Tests performed with different $m$ show a similar behavior.

In Figure 4 we compare two extreme cases where $\alpha$ is near the boundaries of its interval $(0,1)$. The results are obtained with $\alpha=0.01$ on the left and $\alpha=0.99$ on the right. In this test, $m=7$ is fixed. Note that the graphs of $B_{p}$ trace the graphs of the error $M$ very well when $m$ is sufficiently large. Comparing the figures on the left and right and Figure 3, we observe that near $\alpha=1$, the error is smaller than elsewhere. This is compatible with the theoretical estimates, as $B_{p}(\alpha, \eta)$ tends to zero as $\alpha$ tends to one. However, note that the value at which the errors level due to $m$ being too small, is also smaller near $\alpha=1$. This is not reflected in our estimates and is slightly better than their prediction. We therefore cannot conclude whether this trend continues at the limit $\alpha \rightarrow 1$ or not.

In Figure 5 we examine the convergence rate in $m$. The figure shows $M$, given by (5.2), as a function of $m$ and the different graphs are obtained with different values of $p$. The results are obtained with $\delta=10^{-2}$ on the left and $\delta=10^{-3}$ on the right. The dashed line is the graph of $A_{m}$, given by (3.12b), indicating the theoretical estimate (3.15). The relative error shows the expected behavior. For sufficiently large $p$, the error decays exponentially with $m$ and (3.15) predicts the error decay well. 

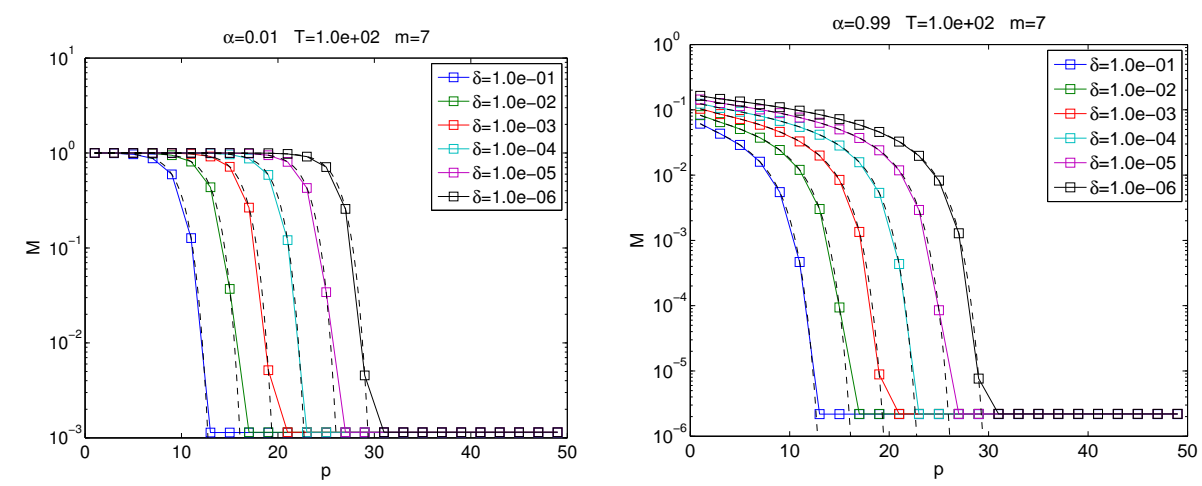

FIG. 4. The maximum value $M$, given by (5.3), of the relative error $\Delta$ on the grid $\tau_{n}$ as a function of $p$; the different graphs correspond to different values of $\delta$; results are obtained with $\alpha=0.01$ on the left and $\alpha=0.99$ on the right.
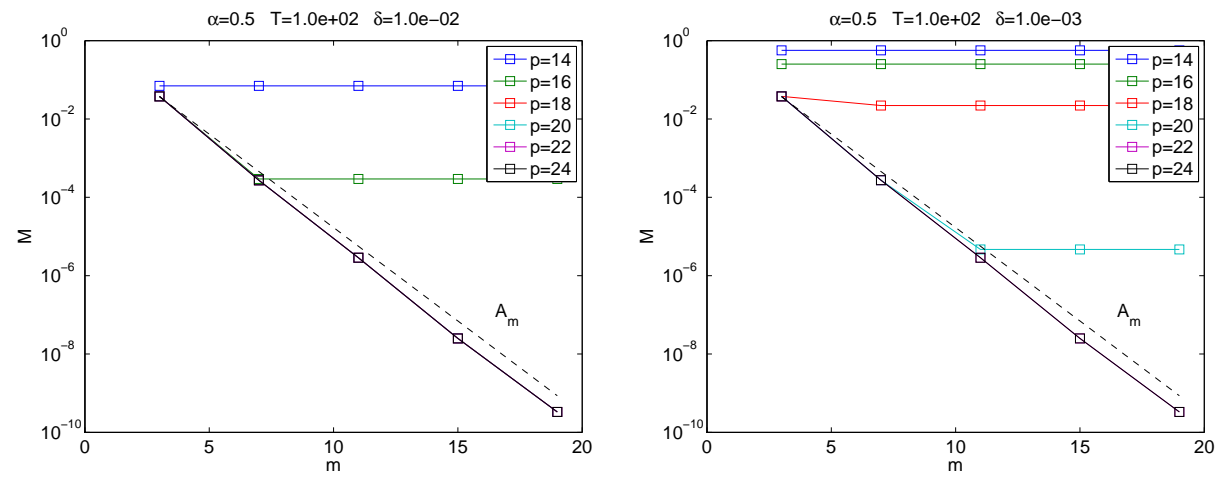

FIG. 5. The maximum value $M$, given by (5.3), of the relative error $\Delta$ on the grid $\tau_{n}$ as a function of $m$; the different graphs correspond to different values of $p$.

5.2. The Mittag-Leffler function. We consider the initial value problem

$$
D^{\alpha} u=-u, \quad u(0)=u_{0}=1,
$$

in $(0, T)$. Its solution is given by

$$
u(t)=E_{\alpha}\left(-t^{\alpha}\right), \quad E_{\alpha}(t)=\sum_{k=0}^{\infty} \frac{t^{k}}{\Gamma(\alpha k+1)} .
$$

Let $t_{n}=n \Delta t$ with $n=0, \ldots, N$, where $\Delta t=T / N$ is the step size and $N \in \mathbb{N}$. The results are obtained with the methods described in section $4.2, \alpha=0.5, T=5$, and $\eta=1 / T=0.2$. Unless mentioned otherwise, the scheme used for approximation of the auxiliary variables is the trapezoidal rule (4.4).

Figure 6 shows the local error $e_{h}=|u-v|$ as a function of time. The results are obtained by discretizing the auxiliary variables by the trapezoidal rule (4.4). The figure shows that as $P$ grows, the error is reduced until it is saturated by the local discretization error. Then, the error can only be reduced by refining the step size.

Figure 7 shows the local error $e_{h}$ as a function of $t$, where the auxiliary variables are discretized by the backward Euler scheme (4.5), showing a similar behavior of the error to that illustrated in Figure 6. Here, however, the errors are larger and become saturated by the discretization error at a significantly smaller $P$. 

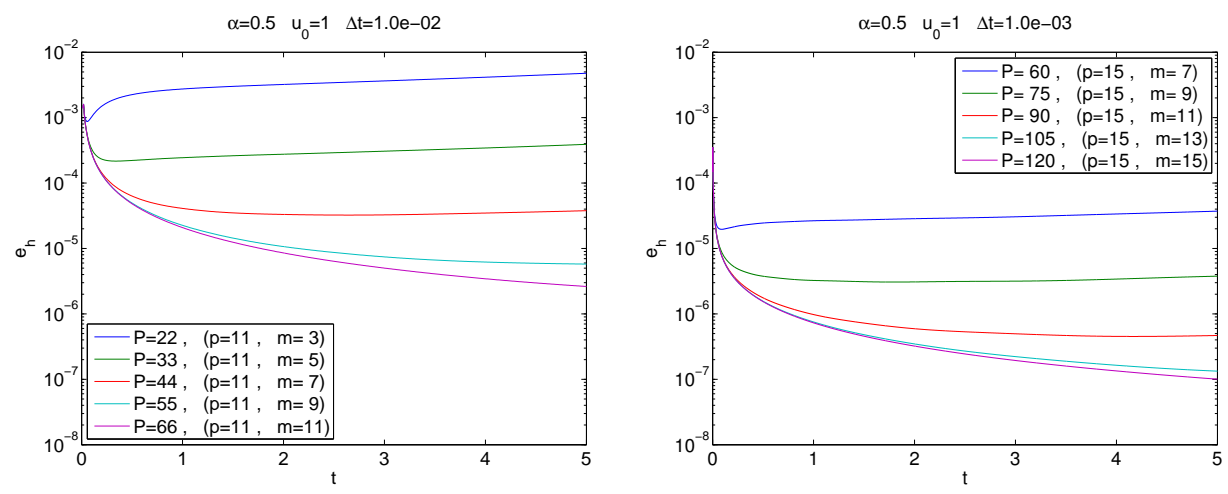

FIG. 6. Accuracy tests for problem (5.4); the local error $e_{h}$ as a function of $t$; auxiliary variables discretized by the trapezoidal rule.
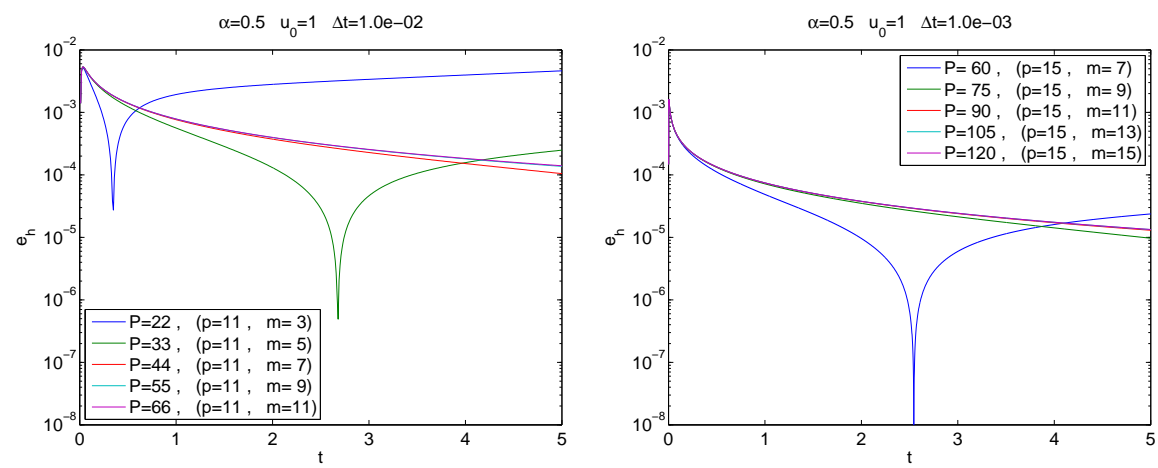

FIG. 7. Accuracy tests for problem (5.4); the local error $e_{h}$ as a function of $t$; auxiliary variables discretized by the backward Euler scheme.

$\alpha=0.5 \quad u_{0}=1$

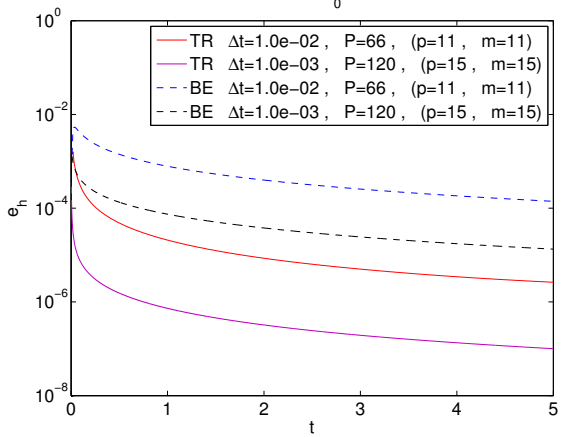

FIG. 8. Accuracy tests for problem (5.4); the local error $e_{h}$ as a function of $t$. A comparison of the errors produced by the two methods.

In Figure 8 we retain only the graphs corresponding to the errors obtained with the highest number of auxiliary variables and compare the results obtained when discretizing the auxiliary variables by the trapezoidal rule (solid lines) and backward Euler scheme (dashed lines).

To test the validity of the theoretical estimates on $p$ and $m$ we set $\Delta t=10^{-4}$ and compute the 2-norm of the error $E=\left\|e_{h}\right\|_{2}$, where

$$
\|v\|_{2}^{2}=\Delta t \sum_{n=0}^{N}\left|v^{n}\right|^{2} .
$$



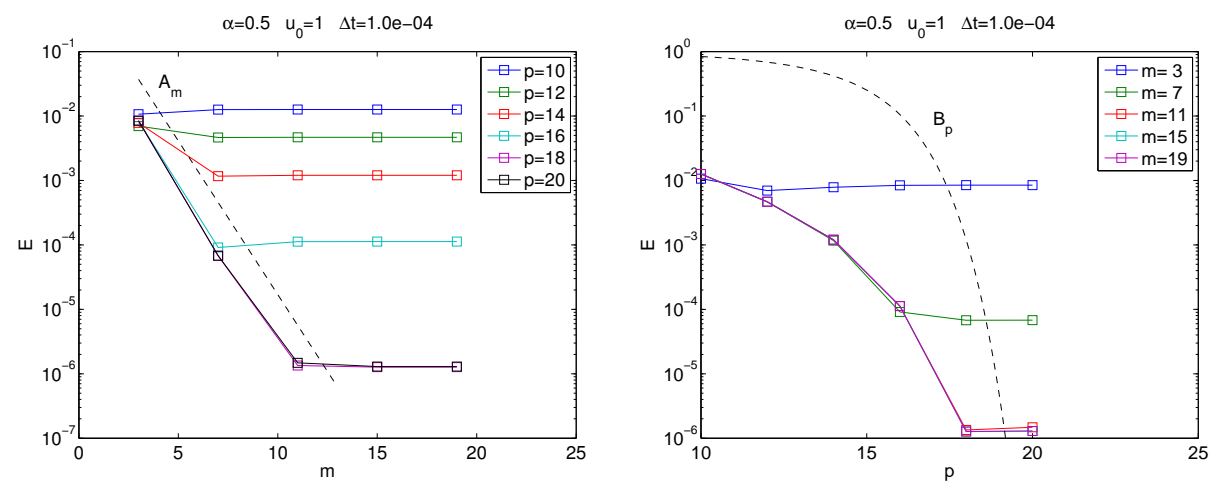

FIG. 9. Accuracy tests for problem (5.4) - verification of estimates (3.14) and (3.15).

Here, $v^{n}$ is the value of the grid function $v$ at $t_{n}=n \Delta t$. Figure 9 shows $\left\|e_{h}\right\|_{2}$ as a function of $m$ and $p$. The dashed lines correspond to the theoretical estimates (3.14) and (3.15). We remark that estimate (3.14) is of the 2-norm of $\left(\mathcal{I}_{\Delta t}-\mathcal{I}_{\Delta t, r}\right) f$, and estimate (3.15) is of the kernel approximation error (both on the continuous level and relative to the approximated quantity), while Figure 9 shows the error of the (discrete) numerical solution. Therefore the results shown in Figure 9 should be compared to estimates derived from a convergence analysis, which may differ from the present estimates. Nevertheless, Figure 9 shows that (3.14) and (3.15) predict the error decay rate well.

5.3. Fractional Van der Pol equation. Consider the nonlinear fractional differential equation

$$
\left(D^{\alpha}\right)^{2} x-\varepsilon\left(1-x^{2}\right) D^{\alpha} x+x=0
$$

in $(0, T)$ with initial conditions

$$
x(0)=x_{0}, \quad D^{\alpha} x(0)=y_{0} .
$$

Here $\varepsilon$ is a nonnegative constant, and $x_{0}, y_{0} \in \mathbb{R}$. For $\alpha=1$, (5.6a) is reduced to the classical Van der Pol equation. In this case it can be shown to have a stable periodic solution. To apply the scheme we write (5.6a) as a system by substituting $y=D^{\alpha} x$. Thus,

$$
\begin{aligned}
D^{\alpha} x & =y, & x(0) & =x_{0}, \\
D^{\alpha} y & =\varepsilon\left(1-x^{2}\right) y-x, & y(0) & =y_{0},
\end{aligned}
$$

in $(0, T)$. Let $t_{n}=n \Delta t$ with $n=0, \ldots, N$, where $\Delta t=T / N$ is the step size and $N \in \mathbb{N}$. In the tests below we fix $\alpha=0.8, \varepsilon=4, T=12$, and $\eta=1 / T$. The scheme used for approximation of the auxiliary variables is the trapezoidal rule (4.4).

First we look at the behavior of the error with respect to the step size. Hence, we choose $p$ and $m$ sufficiently large and compare numerical solutions computed with different $\Delta t$. In this test we use $p=25$ and $m=25$. Figure 10 shows on the left the reference solution computed $\Delta t=10^{-4}$, and on the right, the difference between the reference solution and other numerical solutions computed with different $\Delta t$.

In Figure 11, numerical solutions computed with different parameters $p$ and $m$ are compared to the reference solution computed with $p=25, m=25$, and $\Delta t=10^{-4}$. 

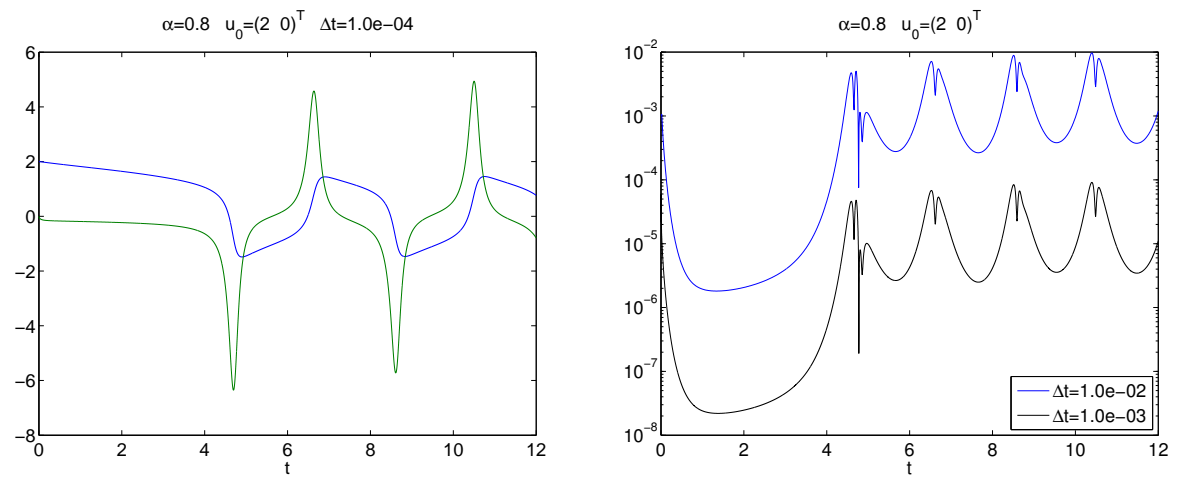

FIG. 10. Fractional Van der Pol equation (5.7); on the left is a reference solution $v_{\text {ref }}$ computed with $p=m=25$ and $\Delta t=10^{-4}$; on the right, numerical solutions computed with $p=m=25$ and different $\Delta t$ are compared with $v_{\text {ref. }}$
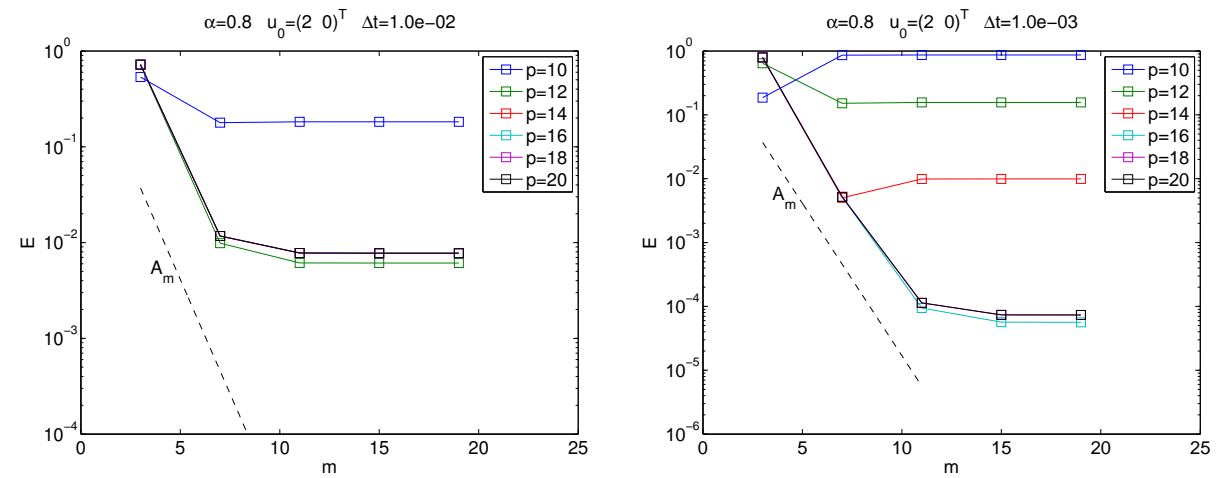

Fig. 11. Fractional Van der Pol equation rm (5.7); numerical solutions computed with $\Delta t=$ $10^{-2}$ (left) and $\Delta t=10^{-3}$ (right) are compared with $v_{\text {ref. }}$ Here $E=\left\|v-v_{\text {ref }}\right\|_{2}$, where $v_{\text {ref }}$ is a reference solution computed with $p=m=25$ and $\Delta t=10^{-4}$.

The figure shows $E=\left\|v-v_{\text {ref }}\right\|_{2}$ as a function of $m$; the different graphs correspond to different $p$.

Finally, we look at how the error of the history term behaves for numerical solutions. To eliminate the discretization error we compare the numerical solutions to reference solutions computed with the same step size but large $p$ and $m$. We repeat the test for two step sizes $\Delta t=10^{-2}$ and $\Delta t=10^{-3}$. The reference solutions are computed with $p=25$ and $m=25$. Figure 12 shows $E$ as a function of $m$; the different graphs correspond to different values of $p$.

6. Concluding remarks. In this work we propose a method for the localization of the convolution (1.1). We present a priori error estimates, showing that for a given time interval $(0, T)$, local time step $\delta>0$, and error tolerance $\varepsilon>0$, the number of terms required to achieve (1.5) is $J=p m$, with $p$ and $m$ satisfying (1.4). Moreover, the estimates, stated by Theorem 3.3, show that the number of poles $J$ required to satisfy a prescribed error tolerance is bounded uniformly for $\alpha \in(0,1)$. In fact, the estimates show that the error term associated with $p$ tends to zero when $\alpha$ tends to one.

We have tested the performance of the scheme approximating the kernel $w_{\delta}$ independently, or as part of a time stepping method. The results are consistent with the theoretical estimates showing the predicted rapid convergence and qualitative behav- 

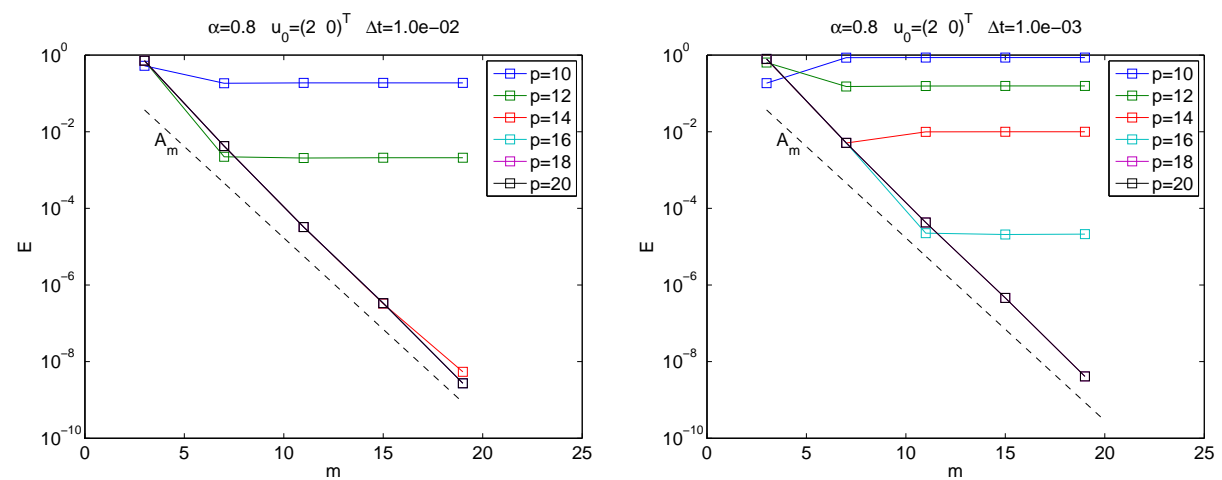

Fig. 12. Fractional Van der Pol equation (5.7); numerical solutions computed with $\Delta t=10^{-2}$ (left) and $\Delta t=10^{-3}$ (right) are compared with $v_{\text {ref. Here }} E=\left\|v-v_{\text {ref }}\right\|_{2}$, where $v_{\text {ref }}$ is a reference solution computed with $p=m=25$ and the same step size as $v$, i.e., $\Delta t=10^{-2}$ on the left and $\Delta t=10^{-3}$ on the right.

ior of the approximation. While our estimates provide a uniform in $\alpha$ bound on the error term associated with $m$, in our numerical tests, near $\alpha=1$ we measure errors smaller than elsewhere. We emphasize that this is not reflected in our estimates, and therefore we cannot conclude whether the error term associated with $m$ vanishes at the limit $\alpha \rightarrow 1$, or not. Nevertheless, the numerical results support the proposition that this term is also bounded uniformly for $\alpha \in(0,1)$.

The incorporation of the kernel compression scheme into a time stepping method involves the introduction of a set of auxiliary variables. In contrast to the methods inspired by [9], where different sets of auxiliary variables are used at different time "windows," here we use a single set of auxiliary variables. That is, in the entire time interval, the same set of auxiliary variables is used. This leads to relatively simple time stepping schemes. We have tested two time stepping schemes incorporating the kernel compression method on two problems: corresponding to the Mittag-Leffler function and a fractional Van der Pol equation. The results illustrate the strength of the method, i.e., its ability to reduce the memory and computational costs. This will be of particular importance for large systems requiring long time integration. For applications, however, methods are required to possess some additional features such as adaptive step size and high-order convergence. We explore some ideas addressing this in [15]. Another topic deserving attention in future work is the study of fully discrete schemes of the type proposed. In particular, the accuracy, stability, and convergence of such schemes should be understood.

Appendix A. Multipole approximation. Below are the proof of Lemma 3.1 and an additional lemma used in the derivation of the relative error estimate. Lemma 3.1, inspired by Lemma 3.5 of [7], states the approximation and provides an error estimate.

Proof of Lemma 3.1. Let

$$
\Phi(z)=\int_{\mathcal{C}}\left|\frac{\rho(\zeta)}{z-\zeta}\right| \mathrm{d}|\zeta| .
$$

Define

$$
\phi=\sum_{k=1}^{p} \phi_{k}, \quad \phi_{k}(z)=\int_{\mathcal{C}_{k}} \frac{\rho(\zeta)}{z-\zeta} \mathrm{d} \zeta,
$$


and similarly, $\Phi=\sum_{k} \Phi_{k}$, and $r=\sum_{k} g_{k}$. Fix some $1 \leq k \leq p$. Owing to the identity

$$
\frac{1}{x-y}=\sum_{s=0}^{m-1} \frac{y^{s}}{x^{s+1}}+\frac{1}{x^{m}} \frac{y^{m}}{x-y},
$$

we have

$$
\phi_{k}(z)=\sum_{s=0}^{m-1} \frac{r_{k}^{s}}{\left(z-c_{k}\right)^{s+1}} Q_{k s}+\left(\frac{r_{k}}{z-c_{k}}\right)^{m} \int_{\mathcal{C}_{k}} \frac{\rho(\zeta)}{z-\zeta}\left(\frac{\zeta-c_{k}}{r_{k}}\right)^{m} \mathrm{~d} \zeta
$$

and

$$
g_{k}(z)=\sum_{s=0}^{m-1} \frac{r_{k}^{s}}{\left(z-c_{k}\right)^{s+1}} \sum_{j=0}^{m-1} \sigma_{k j} \omega^{j s}+\left(\frac{r_{k}}{z-c_{k}}\right)^{m} \sum_{j=0}^{m-1} \frac{\sigma_{k j} \omega^{j m}}{z-\left(c_{k}+r_{k} \omega^{j}\right)} .
$$

Observing that $\omega^{m}=1$ and

$$
\sum_{j=0}^{m-1} \sigma_{k j} \omega^{j s}=Q_{k s}
$$

we find

$$
g_{k}(z)=\sum_{s=0}^{m-1} \frac{r_{k}^{s}}{\left(z-c_{k}\right)^{s+1}} Q_{k s}+\left(\frac{r_{k}}{z-c_{k}}\right)^{m} g_{k}
$$

and thus obtain

$$
\left|\phi_{k}(z)-g_{k}(z)\right| \leq a^{-m}\left|\int_{\mathcal{C}_{k}} \frac{\rho(\zeta)}{z-\zeta}\left(\frac{\zeta-c_{k}}{r_{k}}\right)^{m} \mathrm{~d} \zeta\right|+a^{-m}\left|g_{k}\right| .
$$

Due to

$$
\left|\int_{\mathcal{C}_{k}} \frac{\rho(\zeta)}{z-\zeta}\left(\frac{\zeta-c_{k}}{r_{k}}\right)^{m} \mathrm{~d} \zeta\right| \leq \int_{\mathcal{C}_{k}}\left|\frac{\rho(\zeta)}{z-\zeta}\right| \mathrm{d}|\zeta|=\Phi_{k}(z),
$$

estimate (A.8) yields

$$
\begin{aligned}
a^{m}\left|\phi_{k}(z)-g_{k}(z)\right| & \leq \Phi_{k}(z)+\left|g_{k}(z)\right| \\
& \leq \Phi_{k}(z)+\left|\phi_{k}(z)-g_{k}(z)\right|+\left|\phi_{k}(z)\right|,
\end{aligned}
$$

and finally, by $\left|\phi_{k}(z)\right| \leq \Phi_{k}(z)$, we get

$$
\left|\phi_{k}(z)-g_{k}(z)\right| \leq \frac{2}{a^{m}-1} \Phi_{k}(z),
$$

and thus the conclusion.

The following lemma is useful for estimating the relative error in cases where the contour $\mathcal{C}$ is contained in the negative half line $(-\infty, 0]$.

LEMma A.1. Let

$$
\phi(z)=\int_{0}^{\infty} \frac{\rho(x)}{z+x} \mathrm{~d} x,
$$

where $\rho \geq 0$ is integrable and $0 \neq z \in \mathbb{C}$ with $\operatorname{Re} z \geq 0$. Then, the following inequalities hold:

$$
\frac{1}{\sqrt{2}} \phi(|z|) \leq|\phi(z)| \leq \sqrt{2} \phi(|z|) .
$$


Proof. The upper estimate of $|\phi|$ is due to

$$
|z+x|^{2}=|z|^{2}+x^{2}+2 x \operatorname{Re} z \geq|z|^{2}+x^{2}
$$

and the inequality

$$
\frac{(a+b)^{2}}{2} \leq a^{2}+b^{2}, \quad a, b \geq 0,
$$

which together imply

$$
\frac{1}{|z+x|} \leq \frac{\sqrt{2}}{|z|+x}
$$

To prove the lower estimate of $|\phi|$, we note that

$$
\frac{1}{z+x}=\frac{\bar{z}+x}{|z+x|^{2}}
$$

and thus

$$
|\phi(z)|^{2}=\left|\int_{0}^{\infty} \frac{\rho(x)(a+x)}{|z+x|^{2}} \mathrm{~d} x\right|^{2}+b^{2}\left|\int_{0}^{\infty} \frac{\rho(x)}{|z+x|^{2}} \mathrm{~d} x\right|^{2},
$$

where $a, b \in \mathbb{R}$ are such that $z=a+i b$. Applying inequality (A.14) once more, we recover

$$
|\phi(z)| \geq \frac{1}{\sqrt{2}} \int_{0}^{\infty} \frac{\rho(x)(a+|b|+x)}{|z+x|^{2}} \mathrm{~d} x .
$$

Thus, due to $|z+x| \leq|z|+x$ and

$$
|z+x|=|a+i b+x| \leq a+|b|+x,
$$

we have the conclusion.

Appendix B. Convolutions and the Laplace transform. In this section we provide some general results pertaining convolutions and the Laplace transform. In the following $W^{k, p}$ and $H^{k}$ denote the standard Sobolev spaces. For $\phi \in L^{2}(0, \infty)$, Parseval's relation,

$$
\int_{0}^{\infty} \mathrm{e}^{-2 \eta t}|\phi(t)|^{2} \mathrm{~d} t=\frac{1}{2 \pi} \int_{-\infty}^{\infty}|\widehat{\phi}(\eta+i \xi)|^{2} \mathrm{~d} \xi,
$$

holds.

Lemma B.1. Let $b \in W^{1,1}(0, \infty)$ with $b(0) \neq 0$, and $|\widehat{b}|>0$ in $\operatorname{Re} z \geq 0$, and $f \in H^{1}(0, \infty)$ with $f(0)=0$. There exists a unique $\rho \in L^{2}(0, \infty)$ satisfying

$$
b * \rho=f \quad \text { in }(0, \infty) .
$$

Moreover, there exists a constant $C$, depending only on $b$, such that

$$
\int_{0}^{\infty}|\rho(t)|^{2} \mathrm{~d} t \leq C \int_{0}^{\infty}\left|f^{\prime}(t)\right|^{2} \mathrm{~d} t
$$


Proof. Since $|\widehat{b}|>0$ in $\operatorname{Re} z \geq 0$, we may define

$$
\widehat{\rho}=\frac{\widehat{f}}{\widehat{b}} .
$$

Owing to $f(0)=0, f \in H^{1}(0, \infty)$, and $b \in W^{1,1}(0, \infty)$, we have

$$
\widehat{\rho}=\frac{\widehat{f}^{\prime}}{\widehat{b}^{\prime}+b(0)} .
$$

By the Riemann-Lebesgue lemma, it holds that

$$
\lim _{|\xi| \rightarrow \infty} \widehat{b}(\eta+i \xi)=\lim _{|\xi| \rightarrow \infty} \widehat{b^{\prime}}(\eta+i \xi)=0
$$

for all $\eta \geq 0$. Hence, the denominator of (B.5) is bounded at a positive distance from zero on each line $\operatorname{Re} z=\eta$ with $\eta \geq 0$. Thus, Parseval's relation (B.1) yields the conclusion.

Lemma B.2. Let $T>0$ and $\eta>0$. Suppose $a \in \mathrm{e}^{\eta t} L^{1}(0, \infty), b \in \mathrm{e}^{\eta t} W^{1,1}(0, \infty)$, $b(0) \neq 0,|\widehat{b}|>0$ in $\operatorname{Re} z \geq \eta$, and

$$
|\widehat{a}| \leq|\widehat{b}| \quad \text { on } \quad C(\eta)=\eta+i \mathbb{R} .
$$

Then, for each $u \in L^{2}(0, T)$, the following estimate holds:

$$
\int_{0}^{T} \mathrm{e}^{-2 \eta t}|a * u(t)|^{2} \mathrm{~d} t \leq \int_{0}^{T} \mathrm{e}^{-2 \eta t}|b * u(t)|^{2} \mathrm{~d} t .
$$

Proof. Fix $u \in L^{2}(0, T)$, and suppose $w \in L^{2}(0, \infty)$ satisfies $w=u$ in $(0, T)$. Then, by Parseval's relation (B.1),

$$
\begin{aligned}
\int_{0}^{T} \mathrm{e}^{-2 \eta t}|a * u|^{2} \mathrm{~d} t & \leq \frac{1}{2 \pi i} \int_{C(\eta)}|\widehat{a}(z) \widehat{w}(z)|^{2} \mathrm{~d} z \\
& \leq \frac{1}{2 \pi i} \int_{C(\eta)}|\widehat{b}(z) \widehat{w}(z)|^{2} \mathrm{~d} z=\int_{0}^{\infty} \mathrm{e}^{-2 \eta t}|b * w|^{2} \mathrm{~d} t,
\end{aligned}
$$

and thus

$$
\int_{0}^{T} \mathrm{e}^{-2 \eta t}|a * u|^{2} \mathrm{~d} t \leq \int_{0}^{T} \mathrm{e}^{-2 \eta t}|b * u|^{2} \mathrm{~d} t+\int_{T}^{\infty} \mathrm{e}^{-2 \eta t}|b * w|^{2} \mathrm{~d} t .
$$

Hence we show that for each $\varepsilon>0$, there exists $w \in L^{2}(0, \infty)$ satisfying $w=u$ in $(0, T)$, and

$$
\int_{T}^{\infty} \mathrm{e}^{-2 \eta t}|b * w|^{2} \mathrm{~d} t \leq \varepsilon
$$

We extend $u$ to $(0, \infty)$ by setting $u=0$ in $(T, \infty)$; hence $u \in L^{2}(0, \infty)$. Let $v$ be such that $v=0$ in $(0, T)$ and

$$
v(t)=\widetilde{v}(t-T), \quad t \in(T, \infty),
$$

where $\widetilde{v}$ is the solution to

$$
b * \widetilde{v}=-f+\beta \mathrm{e}^{-\mu \tau} \quad \text { in } \quad(0, \infty)
$$


with $\mu>0$,

$$
f(\tau)=b * u(\tau+T)=\int_{0}^{T} b(\tau+T-s) u(s) \mathrm{d} s,
$$

and $\beta=f(0)$. We note that

$$
f=q * u, \quad q(\tau)=b(\tau+T) \chi_{(0, \infty)}(\tau),
$$

where $q \in \mathrm{e}^{\eta \tau} W^{1,1}(0, \infty)$, and $u \in \mathrm{e}^{\eta t} L^{2}(0, \infty)$, and therefore $f \in \mathrm{e}^{\eta \tau} H^{1}(0, \infty)$. Thus $\widetilde{v} \in \mathrm{e}^{\eta \tau} L^{2}(0, \infty)$, by Lemma B.1. Let $w=u+v$. Owing to the above, we have $w \in \mathrm{e}^{\eta t} L^{2}(0, \infty)$. In addition, due to

$$
b * w(t)=b * u(t)+b * v(t)=\beta \mathrm{e}^{-\mu(t-T)}, \quad t \in(T, \infty),
$$

we recover

$$
\int_{T}^{\infty} \mathrm{e}^{-2 \eta t}|b * w|^{2} \mathrm{~d} t=\frac{|\beta|^{2}}{2} \frac{\mathrm{e}^{-2 \eta T}}{\eta+\mu} .
$$

Observing that the right-hand side of (B.17) tends to zero as $\mu$ tends to infinity, we have the conclusion.

Lemma B.3. Suppose $a, b \in L_{\mathrm{loc}}^{2}[0, \infty)$ are such that for each $T>0$, the estimate

$$
\|a * f\|_{L^{2}(0, T)} \leq\|b * f\|_{L^{2}(0, T)}
$$

holds for all $f \in L^{2}(0, T)$; then

$$
|a(t)| \leq|b(t)| \quad \text { a.e. } t \in(0, \infty) .
$$

Proof. Note that (B.18) holds for all $f \in L^{1}(0, T)$ : Suppose $T>0$ and $f \in$ $L^{1}(0, T)$. Recall that $L^{2}(0, T) \subset L^{1}(0, T)$, and the operators $g \mapsto a * g$ and $g \mapsto b * g$ are continuous from $L^{1}(0, T)$ into $L^{2}(0, T)$. Thus, by approximating $f$ in $L^{1}(0, T)$ by $L^{2}(0, T)$ functions, we recover (B.18).

Let $I=\left[t_{1}, t_{2}\right] \subset[0, \infty)$ be closed and bounded. We substitute

$$
f_{\beta}(t)=\frac{t^{-1+\beta}}{\Gamma(\beta)} \chi_{I}(t), \quad \beta>0,
$$

for $f$ in (B.18) with $T=t_{2}$. Note that, indeed, $f_{\beta} \in L^{1}\left(0, t_{2}\right)$. The following assertions hold: for each $g \in L^{2}(I)$, the limit, $\lim _{\beta \rightarrow 0^{+}} g * f_{\beta}=g$ exists in $L^{2}(I)$, and $a * f_{\beta}=b * f_{\beta}=0$ a.e. in $\left(0, t_{1}\right)$. Due to the above, we obtain

$$
\|a\|_{L^{2}(I)} \leq\|b\|_{L^{2}(I)}
$$

and thus the conclusion, since $I$ is arbitrary.

\section{REFERENCES}

[1] K. Diethelm, N. J. Ford, And A. D. Freed, A Predictor-corrector approach for numerical solution of fractional differential equations, Nonlinear Dynam., 29 (2002), pp, 3-22.

[2] S. B. Yuste ANd L. AcEdo, An explicit finite difference method and a new Von Neumanntype stability analysis for fractional diffusion equations, SIAM J. Numer. Anal., 42 (2005), pp. 1862-1874. 
[3] B. Jin, R. Lazarov, And Z. Zhou, Two fully discrete schemes for fractional diffusion and diffusion-wave equations with nonsmooth data, SIAM J. Sci. Comput., 38 (2016), pp. A146A170.

[4] H. Brunner ANd D. SchötzAu, hp-discontinuous Galerkin time-stepping for Volterra integrodifferential equations, SIAM J. Numer. Anal., 44 (2006), pp. 224-245.

[5] I. Podlubny, Fractional Differential Equations, Academic Press, San Diego, 1999.

[6] N. J. Ford And A. C. Simpson, The mumerical solution of fractional differential equations: Speed versus accuracy, Numer. Algorithms, 6 (2001), pp. 333-346.

[7] B. Alpert, L. Greengard, And T. Hagstrom, Rapid evaluation of nonreflecting boundary kernels for time-domain wave propagation, SIAM J. Numer. Anal., 37 (2000), pp. 11381164.

[8] J.R. LI, A fast time stepping method for evaluating fractional integrals SIAM J. Sci. Comput., 31 (2010), pp. 4696-4714.

[9] C. Lubich AND A. SchäDle, Fast convolution for nonreflecting boundary conditions, SIAM J. Sci. Comput., 24 (2002), pp. 161-182.

[10] A. Schädle, M. López-Fernández, ANd C. Lubich, Fast and oblivious convolution quadrature, SIAM J. Sci. Comput., 28 (2006), pp. 421-438.

[11] M. López-Fernández, C. Lubich, ANd A. Schädle, Adaptive fast and oblivious convolution in evolution equations with memory, SIAM J. Sci. Comput., 30 (2008), pp. 1015-1037.

[12] G. Beylkin and L. Monzón, Approximation by exponential sums revisited, Appl. Comput. Harmon. Anal., 28 (2010), pp. 131-149.

[13] D. Conte And I. Del Prete, Fast collocation methods for Volterra integral equations of convolution type, J. Comput. Appl. Math., 196 (2006), pp. 652-663.

[14] M. López-Fernández, C. Palencia, And A. Schädle, A spectral order method for inverting sectorial Laplace transforms, SIAM J. Numer. Anal., 44 (2006), pp. 1332-1350.

[15] D. BAfFet and J. S. Hesthaven, High-order accurate adaptive kernel compression timestepping schemes for fractional differential equations, J. Sci. Comput., to appear, https: //doi.org/10.1007/s10915-017-0393-z.

[16] M. Abramowitz and I. A. Stegun, Handbook of Mathematical Functions, National Bureau of Standards, Gaithersburg, MD, 1972.

[17] A. ERdÉLYI, Higher Transcendental Functions, Vol. 1, McGraw-Hill, New York, 1953. 